\title{
Phase Space Analysis and Soliton Solution for Quartic Potential
}

\author{
Muzzammil Ahmad Bhat ${ }^{1}$, N. K. Pandey ${ }^{2}$, Shiekh Abid Hussain ${ }^{3}$ \\ Department of Physics Rani Durgavati University Jabalpur \\ Department of Physics Govt. Degree College Tral Kashmir
}

\begin{abstract}
We study dynamics of solitons under the rotating wave approximation by considering a weak quartic nearest neighbour interparticle interaction potential. Also we discussed qualitative analysis of dynamical system in the phase plane where localised solutions correspond to a separatrix curve.
\end{abstract}

Keywords: Solitons, RWA, Separatrix curve

\section{Introduction}

Most natural systems are nonlinear and aretherefore modelled by nonlinear systems of equations. The essential difference between linear and nonlinear systems is that linear systems satisfy a simple superposition principle, that is, any two solutions of a linear system added together form a new solution to the same equations, and this is not the case for solutions of nonlinear systems. This superposition principle allows the solution of a linear problem to be broken into pieces, which are then solved independently by, for example, the Fourier or Laplace transform, and then added back to form a solution to the original problem.

Despite the difficulty caused by the lack of superposition principles, the last 60 years have seen revolutionary progress in solving nonlinear systems, guided by advances in experiments, phenomenal success in the computer simulation of nonlinear systems, and new mathematical analytical tools, such as the inverse spectral transform and methods based on Hamiltonian systems. The synergy between theory, computation, and experimental sciences continues to lead researchers to new levels of understanding. One field of nonlinear science that has experienced some of the most spectacular progress is wave propagation phenomena. In this class of problems, asymptotic procedures that take advantage of small parameters in physical regimes of interest often result in a few "universal" partial differential equations (PDEs). It is one of the mysteries of nature that many of these equations turn out to be integrable, which essentially means that their solutions can be represented as a superposition of special wave modes. Thus some manifestly nonlinear problems can after all be broken down and solved via a nonlinear decomposition in a manner analogous to the way that linear wave equations are solved with Fourier or Laplace transforms. The most famous of these special wave modes are perhaps the solitons, localized waves that collide elastically, suffering only a shift in phase. This robustness of solitons to overcome strong perturbations is largely due to a subtle balancebetween the tendency of nonlinearity to increase the wave slope and the linear dispersion that tends to flatten a wave. The occurrence of this type of balance is widespread in fluid mechanics.

In spite of the great progress made in the theoretical studies, there remain many problems awaiting further development and analysis. Although many suggestions about potential physical realizations of the theoretical findings for the solitons in nonlinear lattices have been put forward in optics, nanophotonics, and matter waves in BECs, most predictions are still awaiting experimental implementation. Thus far, experimental observations that are relevant to nonlinear lattices have been reported only in photorefractive crystals with photo induced lattices, and in photonic-crystal fibers filled with an index-matching liquid. No specific experimental studies of nonlinear lattices have been reported in the realm of BECs, nor in nanophotonics systems, such as nanowire arrays. It is expected that the theoretical predictions that may be most plausible for experimental realization are those involving 1D settings. These include the creation of solitons and their bound states above the predicted existence threshold, the demonstration of their mobility and collisions, the realization of the predicted sp0ontaneous symmetry breaking in nonlinear double-well potentials, etc. An essentially more challenging problem for experimental implementation is the creation of $2 \mathrm{D}$ solitons that may be supported by nonlinear lattices. Ultimately, an entire field waits experimental and theoretical exploration.

Since the work of Sievers and Takeno[1] reported a kind of intrinsic localized modes in a homogenous nonlinear monoatomic chain in 1988, there has been a substantial and increasing interest in the study of such modes for other similar physical systems[2,3], especially for the nonlinear diatomic chain[4,5-9]. Although the diatomic chain is one of the simplest physical models, it is related to many real physical systems such as two-component hydrogen-bonded dimers[10,11], oxidicperovskite ferroelectric crystals (e.g. $\mathrm{SrTiO}_{3}, \mathrm{BaTaO}_{3}$, etc.) along the (100) direction[12], and the Josephson superlattice[13]. Strongly localized anharmonic modes can occur in a perfect diatomic chain when a quartic term is added to the harmonic potential to characterize the nearest-neighbor restoring force between particles[4,6], and the localized modes are different from those of monoatomic chain[14] with the nearest-neighbor interaction nonlinearity.

\section{Model Hamiltonian and Equations of Motion}

The model taken is a $1 \mathrm{D}$ diatomic lattice with a two-body nearest-neighbor interaction potential. The Hamiltonian of the system is given by 


\section{International Journal of Science and Research (IJSR) \\ ISSN (Online): 2319-7064}

Index Copernicus Value (2015): 78.96 | Impact Factor (2015): 6.391

$H=\sum_{i}\left[\frac{1}{2} m_{i}\left(\frac{d u_{i}}{d t}\right)^{2}+V\left(u_{i+1}-u_{i}\right)\right]$

where $u_{i}=u_{i}(r)$ is the displacement from its equilibrium position of the $i^{\text {th }}$ particle with the mass $m_{i}=m \delta_{i, 2 k}+$ $M \delta_{i, 2 k}(M>m, k$ is an integer). The potential $V(r)$ is quite general; typically it can be a standard two-body potential of Toda, Born-Mayer-Coulomb, Lennard-Jones, or Morse type (for their detailed expressions, see Reference [15]). We focus on displacements with smaller amplitude; thus we Taylor expand the potential $V(r)$ at the equilibrium position $r$ $=0$ in a power series of the displacements to fourth order. As a result we have an approximate $K_{2}-K_{3}-K_{4}$ potential

$$
V(r)=\frac{1}{2} K_{2} r^{2}+\frac{1}{3} K_{3} r^{3}+\frac{1}{4} K_{4} r^{4}
$$

Denoting by $s_{n}(t)$ the displacement of atom $n$, the equation of motion of the harmonic atomic chain subjected to a nonlinear force may be written generally in the form

$$
m_{n} \frac{d^{2} u_{n}}{d t^{2}}=k\left(s_{n+1}+s_{n-1}-2 s_{n}\right)+\lambda f\left(s_{n+1}, s_{n-1}, s_{n}\right)
$$

where $k$ is the harmonic coupling constant, $\lambda$ is a parameter of nonlinearity and $f$ is a nonlinear function. We assume the diatomic chain consists of atoms of two masses periodically located, i.e., $m_{n}=m$ for $n=2 j$ and $m_{n}=M$ for $n=2 j+1$.

In the linear case $(\lambda=0)$ model $(\mathbf{2 . 3})$ has two branches of frequency of vibration:

$$
\omega_{ \pm}^{2}=\frac{k}{M m}\left[(M+m) \pm \sqrt{(M-m)^{2}+4 M m \cos ^{2}(a q)}\right]
$$

whereqandaare the wave number and lattice spacing, respectively, and the minus sign corresponds to lowerfrequency acoustic modes and the plus sign to upperfrequency optical modes. At $q=\pi / 2 a$ these two modes are separated by the spectrum gap $\Delta \omega^{2}=\omega_{02}^{2}-\omega_{01}^{2}=$ $2 k(M-m) / M m$, where $\omega_{01}^{2}=2 k / M$ and $\omega_{02}^{2}=2 k / m$ are the gap edge frequencies, and with a cutoff frequency $\omega_{0 m}^{2}=\omega_{01}^{2}+\omega_{02}^{2}=2 k(M+m) / M m$ at $q=0$ due to the discreteness of the system. The gap width is proportional to the mass difference (M-m), so that we expect the properties of soliton solutions to strongly depend on this parameter.

Solutions of a diatomic chain with of quartic nonlinearity:For simplicity, we only consider a weak quartic nearest-neighborinterparticle interaction potential and a quartic substrate potential for the model of the nonlinear diatomic chain. In the former case model (2.3) becomes

$m_{n} \ddot{s}_{n}=k\left(s_{n+1}+s_{n-1}-2 s_{n}\right)+\lambda\left[\left(s_{n+1}-s_{n}\right)^{3}+\left(s_{n-1}-s_{n}\right)^{3}\right]$

We look for the monochromic and stationary solution of the form

$$
s_{n}(t)=\varphi_{n} \cos \omega t,
$$

where $\varphi_{n}$ is the amplitude of the $n$th atom. Therefore equation (2.5) is transformed into

$$
\begin{aligned}
-m_{n} \omega^{2} \varphi_{n}=k\left(\varphi_{n+1}+\varphi_{n-1}-2 \varphi_{n}\right) & \\
& +\left(\frac{3 \lambda}{4}\right)\left[\left(\varphi_{n+1}-\varphi_{n}\right)^{3}+\left(\varphi_{n-1}-\varphi_{n}\right)^{3}\right]
\end{aligned}
$$

under the rotating-wave approximation, i.e., neglecting the higher-harmonic terms.

As has been mentioned above, in the linear limit the motion of the heavy and light atoms is exactly decoupled at $(q=\pi / 2 a)$. Although the nonlinearity breaks the symmetry, we make the following ansatz under the condition of weak nonlinearity according to Refs. $[3,16,17]$

$$
\begin{array}{cc}
\varphi_{n}=(-1)^{j} v_{2 j}, \quad & n=2 j ; \\
\varphi=(-1)^{j} \omega_{2 j+1}, & n=2 j
\end{array}
$$

This means that the particle displacement is split into two fields by introducing different variables for heavy and lightatom oscillations. Then Equation(2.7) can be rewritten as

$$
\begin{aligned}
-\left(m \omega^{2}-2 k\right) v_{n} & =k\left(w_{n}-w_{n-1}\right) \\
& +\left(\frac{3 \lambda}{4}\right)\left[\left(w_{n}-v_{n}\right)^{3}-\left(w_{n-1}+v_{n}\right)^{3}\right], \\
-\left(M \omega^{2}-2 k\right) w_{n} & =k\left(v_{n}-v_{n+1}\right) \\
& +\left(\frac{3 \lambda}{4}\right)\left[\left(v_{n}-w_{n}\right)^{3}-\left(w_{n}+v_{n+1}\right)^{3}\right] .
\end{aligned}
$$

Assuming that both fields $v$ and $w$ are slowly varying in space and time, it appears to be sufficient to keep only the first derivatives. Then Equations (2.9) are reduced to the following system of coupled ordinary differential equations

$$
\begin{aligned}
& \frac{d v}{d x}=-\Delta_{2} w+\beta w\left(w^{2}+3 v^{2}\right), \\
& \frac{d w}{d x}=\Delta_{1} v-\beta v\left(v^{2}+3 w^{2}\right) .
\end{aligned}
$$

where the lattice spacing is set to unity and the parameters are defined as

$$
\Delta_{1}=\frac{\left(m \omega^{2}-2 k\right)}{k}, \quad \Delta_{2}=\frac{\left(M \omega^{2}-2 k\right)}{k}, \quad \beta=\frac{3 \lambda}{2 k}
$$

Equation (2.10) describes the dynamics of a Hamiltonian system with one degree of freedom and the conserved energy

$$
E=-\frac{1}{2}\left(\Delta_{1} v^{2}+\Delta_{2} w^{2}\right)+\frac{\beta}{4}\left(v^{4}+6 v^{2} w^{2}+w^{4}\right),
$$

where functions $w$ and $v$ may be considered as the generalized coordinate and momentum, respectively. Fortunately, Equation (2.10)can be exactly integrated with the help of an auxiliary function[16,17]z=w/v, with which the following general solutions are obtained for the dynamical system (2.10)

$$
\left(\frac{d z}{d x}\right)=\left(\Delta_{1}+\Delta_{2} z^{2}\right)^{2}+4 \beta E\left(z^{4}+6 z^{2}+1\right)
$$




\section{International Journal of Science and Research (IJSR) \\ ISSN (Online): 2319-7064}

Index Copernicus Value (2015): 78.96 | Impact Factor (2015): 6.391

$$
v^{2}=\frac{\left(\Delta_{1}+\Delta_{2} z^{2}\right) \pm \sqrt{\left(\Delta_{1}+\Delta_{2} z^{2}\right)^{2}+4 \beta E\left(z^{4}+6 z^{2}+1\right)}}{\beta\left(z^{4}+6 z^{2}+1\right)} \ldots
$$

We mention that most of the results in this section appeared in references [16,17]. Moreover, fittingpotentials always contain asymmetrical terms in the Taylor expansion near equilibrium. These asymmetrical terms will bring different features to the solution of the diatomic chain and should be treated by different methods.[8,9,18] Through qualitative analysis of dynamical systems in the phase plane $(w, v)$, where the localized solutions correspond to a separatrix curve, different kinds of solutions can be obtained. If the nonlinear parameter is fixed, say $\lambda>0$ (or $>0$ ), then the solutions are characterized bydifferent values of the conserved quantity $E$, as well as of theparameters $\Delta_{1}$ and $\Delta_{2}$.

When $\Delta_{1}, \Delta_{2}<0$, i.e. , $0<\omega<\omega_{1}$, there is only a centre point $v=w=0$ in the phase plane for two dynamical systems. In this case separatrix curves and, subsequently, nontrivial localized solutions are absent. However, when $\Delta_{1}<$ 0 and $\Delta_{2}>0$, i. e., $\omega_{1}<\omega<\omega_{2}$, the system (2.10) has a saddle point at $v=w=0$ and two center points at $\left( \pm \sqrt{\frac{\Delta_{2}}{\beta}}, 0\right)$. As shown in figure 1, the separatrix curve on the phase plane correspond to $E=0$, and equation (2.13) is easily integrated to give the same expression:

$$
z= \pm\left(\left|\Delta_{1}\right| / \Delta_{2}\right)^{1 / 2} \operatorname{coth} y,\left(y=\sqrt{\left|\Delta_{1}\right| \Delta_{2} x}\right)
$$

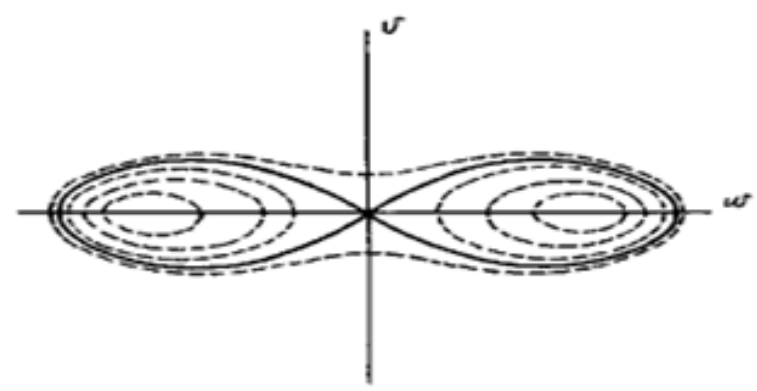

Phase diagram of dynamical system

Substituting this result into (5.12), we have the following localized solution of $v$ and $w$ for system $(\mathbf{2 . 1 0})$ :

$$
\begin{aligned}
& v^{2} \\
& =\frac{2\left|\Delta_{1}\right| \Delta_{2}^{2} \sinh ^{2} y}{\beta\left(\Delta_{1}^{2} \cosh ^{4} y+6\left|\Delta_{1}\right| \Delta_{2} \cosh ^{2} y \sinh ^{2} y+\Delta_{2}^{2} \sinh ^{4} y\right)} \\
& w^{2} \\
& =\frac{2 \Delta_{1}^{2} \Delta_{2} \cosh ^{2} y}{\beta\left(\Delta_{1}^{2} \cosh ^{4} y+6\left|\Delta_{1}\right| \Delta_{2} \cosh ^{2} y \sinh ^{2} y+\Delta_{2}^{2} \sinh ^{4} y\right)} .
\end{aligned}
$$

looking at the behavior of solution (2.17) near the bottom of the linear spectrum gap we find, Near the gap bottom $\omega=\omega_{1}+\epsilon(0<\epsilon \ll 1)$, the parameter $\Delta_{2} \sim \epsilon$, neglecting $\epsilon^{2}$ terms in (2.17), the amplitudedistribution of vibration of heavy atoms for the solution tends to

$$
w=\left(2 \in /_{\beta}\right)^{1 / 2} \operatorname{sech}\left(\sqrt{\left|\Delta_{1}\right| \in x}\right),
$$

which is a standard form of the single stationary nonlinear Schrodinger soliton. This kind of localized vibration of particles with frequency within the forbidden band of the linear spectrum is called a gap mode or gap soliton and is essentially a nonlinear effect.

\section{Conclusion}

In this work, we have studied vibrations of a pure onedimensional crystal lattice with (positive) quartic anharmonicity by paying particular attention to localized modes with frequencies above the harmonic frequency band. We have studied the concept of anharmonicself localized modes in much wider areas in one-dimensional lattice as compared with the previous studies, where only the quartic anharmonicity was taken care of $18,19,20,21,23]$. The theory was formulated by making extensive use of the Lattice-Green's function method which provides us with a natural theoretical basis of studying the localization properties of anharmonic vibrations and for justifying the use of the so called rotating wave approximation RWA.

\section{References}

[1] A. J. Sievers and S Takeno, Phys. Rev. Lett. 61, 970 (1988).

[2] O. Cardoseel al., Phys. Rev. Lett. 65, 1869 (1990).

[3] O. A. Chubykalo, Phys. Lett. A 189, 403 (1994).

[4] O.A. Chubykalo, A.S. Kovalev, and O.V. Usatenko, PhysRev. B 47, 31531993.

[5] Y. S. Kivshar and N. Flytzanos, Phys. Rev. A 46, 7972 (1992).

[6] O. A. Chubykalo and Y. S. Kivshar, Phys. Rev. E 48, 4128 (1993).

[7] A. Champaet al., Phys. Rev. B 48, 10169 (1993).

[8] J. M. Bilbaultet al., Phys. Rev B 47, 5748, (1993).

[9] S. A. Kiselevet al., Phys. Rev. B 48, 13508 (1993); 50, 9135 (1994).

[10] J. F. Nagle et al., J. Chem. Phys. 72, 3959 (1980).

[11] Y. P. Mei et al., Phys. Rev. B 48, 577 (1993).

[12] H. Buttner and H. Biltz, in Solitons and Condensed Matter Physics, edited by A. R. Bishop and T. Schneider (Springer-Verlag, Berlin, 1978), P. 162.

[13] D. Barday and M. Remoissnet, Phys. Rev. B 41, 10387 (1990); 43, 7297 (1991).

[14] O. A. Chubykalo and Y. S. Kivshar, Phys. Lett. A 178, 123 (1993).

[15] S. Jimenez and V. V. Konotop, Phys. B 60, 6465 (1999).

[16] Y.S. Kivshar and N. Flytzanis, Phys. Rev. A 46, 7972 1992.

[17] O.A. Chubykaloet al., Phys. Rev. B 47, 3153 (1993).

[18] A. Campaet al., Phys. Rev. B 48, 10169 (1993).

[19] Roberto Camassa, James M. Hyman , Benjamin E LucePhysica D 123 (1998) 1-20.

[20] C. Doering, X. Wang, Attractor dimension estimates fortwo-dimensional shear flows, Physica D 123 (1998) 206-222.

[21] M.J. Ablowitz, J.E Ladik, Nonlinear difference schemesand inverse scattering, Studies Appl. Math. 55 (3) (1976)213-229.

[22] Sen-yue Lou and Guoxiang Huang, Mod. Phys. Lett. A 9,1231 1995.

[23] M.A. Bhat, et al. Soliton solution in nonlinear lattice with nearest neighbour Born Mayer interaction, J.Taibah Univ. Sci. (2016),http://dx.doi.org/10.1016/j.jtusci.2016.02.005

\section{Volume 6 Issue 1, January 2017

